\title{
Seroepidemiology of human Toxoplasma gondii infection in China
}

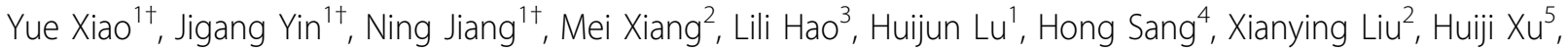
Johan Ankarklev', Johan Lindh ${ }^{6}$, Qijun Chen ${ }^{1,3,6^{*}}$

\begin{abstract}
Background: Toxoplasmosis is an important zoonotic parasitic disease worldwide. In immune competent individuals, Toxoplasma gondii preferentially infects tissues of central nervous systems, which might be an adding factor of certain psychiatric disorders. Congenital transmission of $T$. gondii during pregnancy has been regarded as a risk factor for the health of newborn infants. While in immune-compromised individuals, the parasite can cause life-threatening infections. This study aims to investigate the prevalence of $T$. gondii infection among clinically healthy individuals and patients with psychiatric disorders in China and to identify the potential risk factors related to the vulnerability of infection in the population.
\end{abstract}

Methods: Serum samples from 2634 healthy individuals and 547 patients with certain psychiatric disorders in Changchun and Daqing in the northeast, and in Shanghai in the south of China were examined respectively for the levels of anti-T. gondii IgG by indirect ELISA and a direct agglutination assay. Prevalence of $T$. gondii infection in the Chinese population in respect of gender, age, residence and health status was systematically analyzed.

Results: The overall anti-T. gondii IgG prevalence in the study population was $12.3 \%$. In the clinically healthy population $12.5 \%$ was sero-positive and in the group with psychiatric disorders $11.3 \%$ of these patients were positive with anti-T. gondii lgG. A significant difference $(P=0.004)$ was found between male and female in the healthy population, the seroprevalence was $10.5 \%$ in men versus $14.3 \%$ in women. Furthermore, the difference of T. gondii infection rate between male and female in the 20-19 year's group was more obvious, with $6.4 \%$ in male population and $14.6 \%$ in female population.

Conclusion: A significant higher prevalence of T. gondii infection was observed in female in the clinically healthy population. No correlation was found between $T$. gondii infection and psychiatric disorders in this study. Results suggest that women are more exposed to $T$. gondii infection than men in China. The data argue for deeper investigations for the potential risk factors that threat the female populations.

\section{Background}

T. gondii is an intracellular parasite that can infect almost all mammals and the importance of this parasite in food safety, human health and animal husbandry has been well recognized. Although the parasites remain dormant in people with normal immune competence, they do pose threats to individuals who are immunocompromized. Examples could be patients with AIDS or organ transplantation [1-3]. It has been estimated that up to one third of the world's population has been

\footnotetext{
* Correspondence: Qijun.Chen@mtc.ki.se

† Contributed equally

'Key Laboratory of Zoonosis, Ministry of Education, Jilin University, Xi An Da Lu 5333, Changchun 130062, PR China
}

infected by $T$. gondii with endemicity from around $10 \%$ to $70 \%[2,4,5]$. Further more, up to $14.8 \%$ of AIDS patients in Southeast Asia region were reported with toxoplasmosis in the central nervous systems [6]. Both primary and re-infection during pregnancy are high risk factors especially to the foetus $[7,8]$. Thus surveillance of $T$. gondii infection and the distribution of the oocysts in the living environments have been regarded as an important measure to prevent the disease.

Due to the fact that $T$. gondii mainly infects cells in the brain of immune competent individuals, it has been postulated that certain psychiatric disorders might be a link to the concurrent infection [9-11]. In several studies, patients with schizophrenia were found to have a 
higher tendency of $T$. gondii infection [12-14], but there has been no conclusive correlation between $T$. gondii infection and psychiatric disease [15].

In this study, we investigated the prevalence of anti- $T$. gondii IgG in the sera of more than 3000 Chinese individuals living in the southern and northern regions of China, among which, a panel of 547 serum samples were collected from patients with certain psychiatric disorders. It was found that the general infection rate of $T$. gondii in the studied population was higher than recorded by the Chinese Ministry of Health, and importantly, results suggest that women have a higher risk of being infected by $T$. gondii than men in China. The data argues for more attention for prevention of $T$. gondii infection in the female population and in particular pregnant women.

\section{Methods}

Study populations and Serum samples

2634 serum samples from clinically healthy individuals were collected in Changchun, Daqing and Shanghai areas (with a population of more than 100 million) from July 2006 to June 2008 (Table 1). 547 serum samples were collected from patients with psychiatric disorders (including Schizophrenia, mania, depression and severe stress) in Changchun regions (Table 1). The age of the studied population spanned from 15 to 65 yeas of age. The study was carried out with permission from the Ethical Committee of Institute of Zoonosis, Jilin University, China (Ref number 20070106). The sera were collected with agreement from the volunteers or patients. Written consensus was obtained from parents of all participating teenagers.

\section{Antigen}

T. gondii tachyzoites (RH strain) were routinely maintained by cultivation in BHK (baby hamster kidney) cell lines as described earlier [16]. Briefly parasites from freshly lysed host cells were harvested, washed in PBS and disseminated by sonication. The cell debris was eliminated by centrifugation and the soluble antigens were collected and diluted to a final concentration of 1 $\mathrm{mg} / \mathrm{ml}$ in PBS for the serological test.

\section{Serological assay}

The Toxoplasma-specific IgG antibodies in 3181 serum samples were tested by Indirect Enzyme Linked Immunosorbant Assay (ELISA) [17] and the results were further verified by direct agglutination test with the Toxo-Screen DA (BioMerieux, France) kit. Briefly, Maxisor micro-ELISA plates (Nalge Nunc International, IL, USA) were coated with $50 \mu \mathrm{l}$ per well of the $T$. gondii antigen $(5 \mu \mathrm{g} / \mathrm{ml})$ at $4^{\circ} \mathrm{C}$ overnight. $100 \mu \mathrm{l}$ of each serum sample diluted at 1:50 was added to the wells in triplicates. Alkaline phosphatase-labeled goat antihuman IgG (Sigma, St. Louis, MO, 1:2000 dilution) and NPP [4-Nitrophenyl phosphate disodium salt hexahydrate] (Sigma, St. Louis, MO) were used to detect the antigen-antibody reaction. The plates were finally read in a Biotek 93 micro-ELISA auto reader 808 at $405 \mathrm{~nm}$. Negative control wells with a negative serum verified by direct agglutination test were included in every plate. The cut-off point of OD values of a positive sample was set to be at least two times higher than that of the negative samples at any dilution point. Finally all sera with positive reactivity in ELISA were further tested with the commercial agglutination kit (Toxo-Screen DA, BioMérieux, France), according to the protocol provided by the manufacturer.

\section{Statistical Analysis}

Results were analyzed with SPSS 15.0 software package. Chi square test was used to analyze the anti-T. gondii IgG seroprevalence in respect of gender, age, residence and psychiatric disorders of the populations. The differences were considered to be statistically significant when the $P$ value was less than 0.05 . Logistic regression analysis was used to assess the association with gender, age, residence and psychiatric disorders of the subjects and T. gondii infection in populations. Adjusted odds ratio (OR) and $95 \%$ confidence interval $(\mathrm{CI})$ were calculated by multivariate analysis using logistic regression.

\section{Results}

The overall prevalence of anti-T. gondii IgG in the studied population was $12.3 \%$. In the clinically healthy group the prevalence was $12.5 \%$ meanwhile $11.3 \%$ in the group containing psychiatric patients (Table 1 and 2 ).

Table 1 Number of serum samples from study regions, gender distribution and seroprevalence.

\begin{tabular}{|c|c|c|c|c|c|c|}
\hline \multirow[b]{2}{*}{ Region (Sample nr) } & \multicolumn{2}{|c|}{$\begin{array}{l}\text { Clinically healthy persons } \\
\text { (nr of positive sera) }\end{array}$} & \multirow[t]{2}{*}{ P-value } & \multicolumn{2}{|c|}{$\begin{array}{l}\text { Psychiatric patients } \\
\text { (nr of positive sera) }\end{array}$} & \multirow[t]{2}{*}{ P-value } \\
\hline & Female & Male & & Female & Male & \\
\hline Changchun (1530) & $536(75)$ & $447(47)$ & 0.1 & $319(31)$ & $228(31)$ & \\
\hline Daqing (758) & $485(64)$ & $273(23)$ & 0.048 & - & - & \\
\hline Shanghai (893) & $294(49)$ & $599(70)$ & 0.04 & - & - & \\
\hline Seroprevalence (\%) & 14 & 10.7 & 0.004 & 9.7 & 13.6 & 0.429 \\
\hline
\end{tabular}


Table 2 Number of psychiatric patients and seroprevalence.

\begin{tabular}{lll}
\hline Disorders & Patient number & $\begin{array}{l}\text { Number of patients } \\
\text { with } \boldsymbol{T} \text {. gondii IgG } \\
\text { and prevalence (\%) }\end{array}$ \\
\hline Schizophrenia & 291 & $26(8.9)$ \\
Mania & 49 & $5(10.2)$ \\
Depressive episode & 29 & $4(13.8)$ \\
$\begin{array}{l}\text { Dissociative disorder } \\
\text { Severe stress and }\end{array}$ & 28 & $3(10.7)$ \\
$\begin{array}{l}\text { adjustment disorders } \\
\text { Obsessive-compulsive }\end{array}$ & 15 & $3(20)$ \\
disorder & 12 & $3(25)$ \\
$\begin{array}{l}\text { Other unspecified } \\
\text { mental disorders }\end{array}$ & 123 & $18(14.6)$ \\
\hline Total & 547 & $62(11.3)$ \\
\hline
\end{tabular}

No correlation between $T$. gondii infection and psychiatric disorders was found. A significant observation was the difference in seroprevalence between male and female groups where $10.5 \%$ of the males and $14.3 \%$ of the females were $T$. gondii positive $(\mathrm{P}=0.004)$ (Table $1)$. The general seroprevalence of individuals living in Changchun, Daqing and Shanghai regions were 12.4\%, $11.5 \%$, and $13.3 \%$ respectively. Significant differences in prevalence between male and female were found in the populations of Daqing in the north and Shanghai in the south, where the prevalences were $8.4 \%$ versus $13.2 \%$ ( $\mathrm{P}$ $=0.048)$ and $11.78 \%$ versus $16.7 \%(\mathrm{P}=0.04)$ respectively. The prevalence in Changchun was $10.5 \%$ and $14.0 \%$ respectively in men and women $(\mathrm{P}=0.1)$.

Sera which had anti- $T$. gondii antibodies were divided into 5 groups based on the age $(<20,20-29,30-39,40-49$ and $>50$ years) of the individuals. The seroprevalence of the five age groups were $11.2 \%, 11.8 \%, 12.6 \%, 13.6 \%$ and $12 \%$ respectively in the clinically healthy population. The seroprevalence in the corresponding age groups of the psychiatric patients were $9.1 \%, 12.8 \%, 7 \%, 13.1 \%$ and $12.9 \%$ respectively. The difference in infection rate among the sample groups was not statistically significant. However, when comparing the seroprevalence adjusted by age, the infection rates in women and men of the 2030 years old group were $14.6 \%$ and $6.4 \%$ respectively. The difference was significant $(P=0.006)$. The prevalence of T. gondii infection in individuals living in urban and rural areas in the northern parts of China (including Changchun and Daqing) were $13.1 \%$ and $9.7 \%$.

The optical density at $405 \mathrm{~nm}$ (OD 405), indicated the amount of specific IgG antibodies in the sera, was also measured in the different samples. No significant differences were observed considering the mean values of all studied groups (Figure 1).

In the logistic regression analysis, ten variables (including age, living location, profession, physical

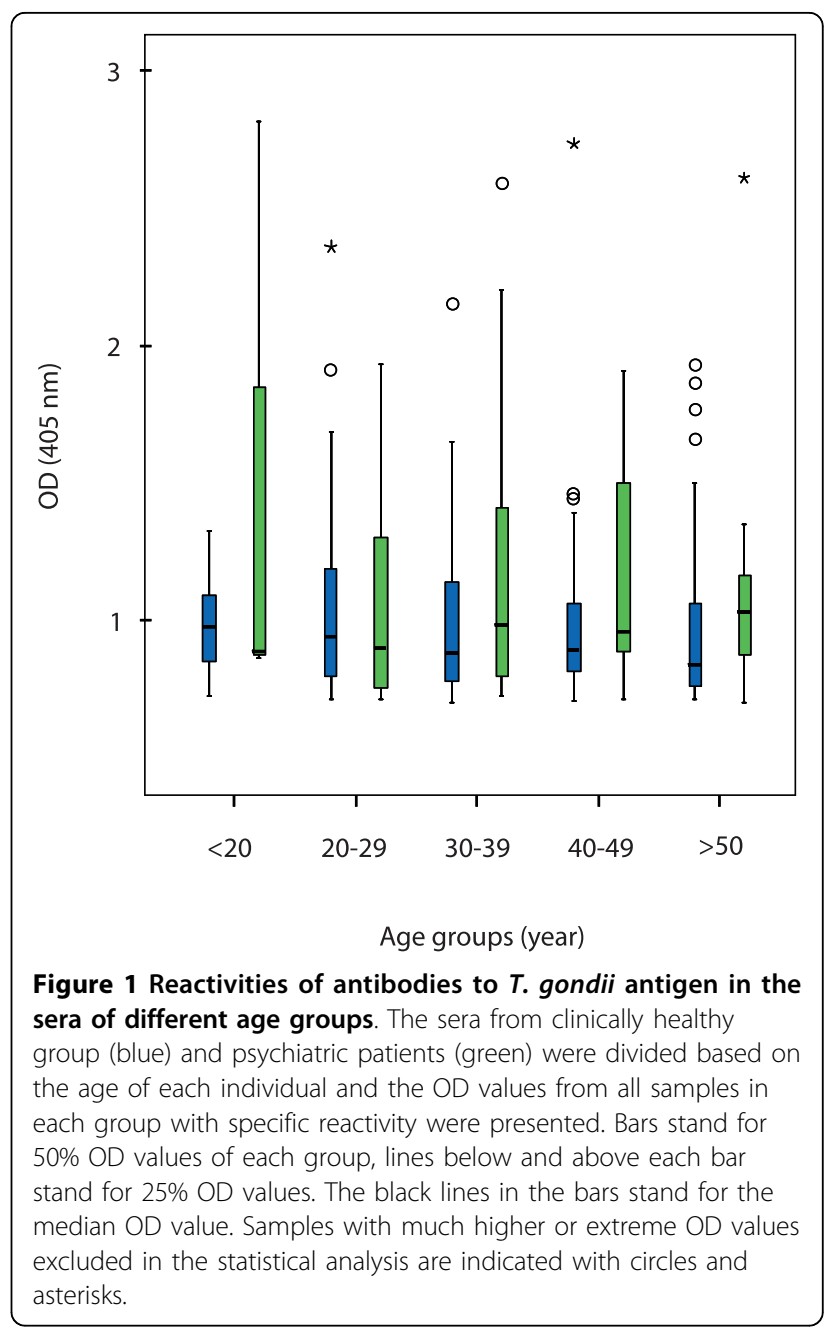

condition and so on) were investigated for potential factors associated with $T$. gondii infection. The results revealed that the only factor associated with $T$. gondii infection was gender (OR $=0.712$; 95\% CI: 0.564-0.899). The rest of the factors did not show any association with $T$. gondii infection.

All positive and some negative samples investigated were verified by agglutination test using the commercially available Toxo-Screen DA kit (BioMérieux, France) as controls. No disagreement between ELISA and DA method was observed (data not shown).

\section{Discussion}

Seroprevalence of $T$. gondii infection in humans and animals including chickens has been used as an indicator of the endemicity of the parasite [18-21]. The epidemiology of toxoplasmosis in many countries has been investigated, but the prevalence of $T$. gondii in the Chinese population has not been clear. During the two national-wide surveys carried out in 1995 and 2004 [22], 
the prevalence of $T$. gondii infection in the Chinese population was reported to be around 7\%. But the number of samples enrolled in the two surveys was limited. In the current study, we investigated more than 3000 individuals living both in the south and the north of the country. Furthermore, the number of serum samples analyzed from male and female individuals was similar (Table 1). We also investigated the sera prevalence of $T$. gondii-specific IgG in more than 500 patients with psychiatric disorders, with the aim of finding a possible correlation between $T$. gondii infection and certain psychiatric symptoms as reported by others [8-15]. The overall infection rate of $T$. gondii in the studied population in China was $12.3 \%$, which was generally lower than that in other countries [1,2]. Earlier studies suggest that the infection rate of free-range chickens in the northeast part of China were 34.5\% [23]. Furthermore, it has recently been reported that cat infection rate of $T$. gondii in China is as high as $79.4 \%$ [24]. The low prevalence in humans found here could be due to the fact that the habit of consuming raw or undercooked meat and having pet animals still not very popular in China. No significant difference in infection rate between individuals living in urban and rural areas in the North part of China was found, which indicated that T. gondii infection is independent of living places. Thus consuming well-cooked meat and proper handling and disposal of faecal material from pet cats are important measures of disease prevention.

The finding that seroprevalance of anti-T. gondii IgG in the females is higher than in the males in the Chinese population is significantly important to the public health. The data indicates that females are at higher risk of contraction $T$. gondii infection in China. This is undoubtedly due to many factors. First, women traditionally take more care of pet animals including cats at home, and secondly, women handle raw meat more frequently than men due to the fact that they spend more time cooking at home. In a recent study with sera from pregnant women living in northeastern China, it was indeed found that taking care of pet animals and consuming raw meat were two main risk factors related to T. gondii infections [5]. The reason that individuals under the age of 20 showed similar infection rate to others could be due to the fact that they tend to play more with animals or due to other unidentified factors. Though it is rare that parasites are reactivated during gestation, infection during pregnancy does pose threat to the life of fetuses and newborns. Thus knowledge of disease prevention is more important in public health programs, especially to the female groups.

In immune competent individuals, $T$. gondii infects a variety of cells of the central nervous tissues, thus the correlation between $T$. gondii infections and certain psychiatric symptoms, especially Schizophrenia, has drawn attention in recent years [10-14]. It has been speculated that parasite components released from the infected brain cells might has effect on surrounding cells and hence impact the mode or behavior of the host [11]. Several studies including a literature surveys supported the conclusion that $T$. gondii infection is modestly associated with Schizophrenia syndrome [15]. However, no correlation between $T$. gondii infection and Schizophrenia was found in this study $(\mathrm{n}=291$, Table 2$)$. Interestingly, the infection rate in the study group was only $8.9 \%$ which was lower than the average rate in the whole population. The patient groups with severe distress and adjustment disorders and Obsessive-compulsive disorder had serum positivity of $20 \%$ and $25 \%$ respectively. However, the figures are not conclusive due to the lower number of patients in the two groups. Unlike previous report, no correlation between $T$. gondii infection and psychiatric disorders were found in this study. This could be due to the genetic background of the Chinese population or the general low infection rate.

\section{Conclusions}

The study has shown that the general infection rate of T. gondii in the Chinese population was $12.3 \%$. Women have a significantly higher risk of being infected by $T$. gondii than men, possibly due to more exposure to the infective sources. No correlation between $T$. gondii infection and psychiatric disorders was observed.

\section{Acknowledgements}

This study was carried out with supports to Q. Chen from the Young Distinguished Scientist Grant (30625029) of the Chinese Natural Scientific Foundation; the grants to invited professor from Jilin University and the Chinese Academy of Medical Sciences, China.

\section{Author details}

${ }^{1}$ Key Laboratory of Zoonosis, Ministry of Education, Jilin University, Xi An Da Lu 5333, Changchun 130062, PR China. ${ }^{2}$ The Second Hospital of Jilin University, Ziqiang Street 218, Changchun 10041, PR China. ${ }^{3}$ Institute of Pathogen Biology, Chinese Academy of Medical Sciences, Beijing, Dong Dan San Tiao, Beijing 100730, PR China. ${ }^{4}$ The Sixth Hospital of Changchun City, North Round Road 4596, Changchun 130040, PR China. ${ }^{5}$ Changzheng Hospital, Shanghai, Fengyang Road 415, Shanghai 200003, PR China. ${ }^{6}$ Department of Parasitology, Mycology and Environmental Microbiology, Swedish Institute for Infectious Disease Control, Nobels väg 18, 17182 Solna, Sweden.

\section{Authors' contributions}

$Y X, J Y, N J, M X, L H, H L, H S, X L$ and $H X$ participated in serum collection and performed the assay. JA, JL provided the antigen. QC planed and supervised the study. All authors contributed to the writing of the manuscript.

\section{Competing interests}

The authors declare that they have no competing interests. 


\section{References}

1. Tenter AM, Heckeroth AR, Weiss LM: Toxoplasma gondii: from animals to humans. Int J Parasitol 2000, 30:1217-1258.

2. Montoya JG, Liesenfeld O: Toxoplasmosis. Lancet 2004, 363:1965-1976.

3. Dubey JP: The history of Toxoplasma gondii-the first 100 years. J Eukaryot Microbiol 2008, 55:467-475.

4. Alvarado-Esquivel C, Torres-Castorena A, Liesenfeld O, García-López C, Estrada-Martínez S, Sifuentes-Álvarez A, Marsal-Hernández J, Esquivel-Cruz R, Castañeda A, Dubey JP: Seroepidemiology of Toxoplasma gondii infection in pregnant women in rural Durango, Mexico. J Parasitol 2009, 95:271274.

5. Liu Q, Wei F, Gao S, Jiang L, Lian H, Yuan B, Yuan Z, Xia Z, Liu B, Xu X, Zhu XQ: Toxoplasma gondii infection in pregnant women in China. Trans $R$ Soc Trop Med Hyg 2009, 103:162-166.

6. Nissapatorn V: Lessons learned about opportunistic infections in Southeast Asia. Southeast Asian J Trop Med Public Health 2008, 39:625-641.

7. Elsheikha EM: Congenital toxoplasmosis: Priorities for further health promotion action. Public Health 2008, 122:335-353.

8. Kravetz JD, Federman DG: Toxoplasmosis in pregnancy. Am J Med 2005, 118:212-216.

9. Walker M, Zunt JR: Parasitic central nervous system infections in immunocompromised hosts. Clin Infect Dis 2005, 40:1005-1015.

10. Torrey EF, Yolken RH: Toxoplasma gondii and schizophrenia. Emerg Infect Dis 2003, 11:1375

11. Carruthers VB, Suzuki Y: Effects of Toxoplasma gondii infection on the brain. Schizophr Bull 2007, 33:745-751.

12. Alvarado-Esquivel C, Alanis-Quiñones OP, Arreola-Valenzuela MA, RodríguezBriones A, Piedra-Nevarez LJ, Duran-Morales E, Estrada-Martínez S, MartínezGarcía SA, Liesenfeld O: Seroepidemiology of Toxoplasma gondii infection in psychiatric inpatients in a northern Mexican city. BMC Infect Dis 2006 6:178.

13. Hinze-Selch D, Däubener W, Eggert L, Erdag S, Stoltenberg R, Wilms S: A controlled prospective study of Toxoplasma gondii infection in individuals with schizophrenia: beyond seroprevalence. Schizophr Bull 2007, 33:782-788.

14. Yolken RH, Bachmann S, Ruslanova I, Lillehoj E, Ford G, Torrey EF, Schroeder J: Antibodies to Toxoplasma gondii in individuals with forstepisode schizophrenia. Clin Infect Dis 2001, 32:842-844.

15. Torrey EF, Bartko JJ, Lun ZR, Yolken RH: Antibodies to Toxoplasma gondii in patients with schizophrenia: a meta-analysis. Schizophr Bull 2007, 33:729-736.

16. Lindström I, Kaddu-Mulindwa DH, Kironde F, Lindh J: Prevalence of latent and reactivated Toxoplasma gondii parasites in HIV-patients from Uganda. Acta Trop 2006, 100:218-222.

17. Balsari A, Poli G, Molina V, Dovis M, Petruzzelli E, Boniolo A, Rolleri E: ELISA for Toxoplasma antibody detection: a comparison with other serodiagnostic tests. J Clin Pathol 1980, 33:640-643.

18. Jones JL, Kruszon-Moran D, Wilson M, Navin TR, Gibbs R, Schulkin J: Toxoplasma gondii infection in the United States: seroprevalence and risk factors. Am J Epidemiol 2001, 154:357-365.

19. Studenicová C, Bencaiová G, Holková R: Seroprevalence of Toxoplasma gondii antibodies in a healthy population from Slovakia. Eur J Int Med 2006, 17:470-473.

20. Sousa OE, Saenz RE, Frenkel JK: Toxoplasmosis in Panama: a 10-year study. Am J Trop Med Hyg 1988, 38:315-322.

21. Ades AE, Parker S, Gilbert R, Tookey PA, Berry $T$, Hjelm M, Wilcox AH, Cubitt D, Peckham CS: Maternal prevalence of Toxoplasma antibody based on anonymous neonatal serosurvey: a geographical analysis. Epidemiol Infect 1993, 110:127-33.

22. $\mathrm{Xu} L Q$, Chen $Y D$, Sun FH: A national survey on current status of the important parasitic diseases in human population. Chin J Parasitol Parasit Dis 2005, 23:332-339.

23. Zhu J, Yin J, Xiao Y, Jiang N, Ankarlev J, Lindh J, Chen Q: A seroepidemiological survey of Toxoplasma gondii infection in free-range and caged chickens in northeast China. Vet Parasitol 2008, 158:360-363.

24. Dubey JP, Zhu XQ, Sundar N, Zhang H, Kwok OC, Su C: Genetic and biologic characterization of Toxoplasma gondii isolates of cats from China. Vet Parasitol 2007, 145:352-356.

\section{Pre-publication history}

The pre-publication history for this paper can be accessed here:http://www. biomedcentral.com/1471-2334/10/4/prepub

doi:10.1186/1471-2334-10-4

Cite this article as: Xiao et al:: Seroepidemiology of human Toxoplasma gondii infection in China. BMC Infectious Diseases 2010 10:4.
Publish with Bio Med Central and every scientist can read your work free of charge

"BioMed Central will be the most significant development for disseminating the results of biomedical research in our lifetime. "

Sir Paul Nurse, Cancer Research UK

Your research papers will be:

- available free of charge to the entire biomedical community

- peer reviewed and published immediately upon acceptance

- cited in PubMed and archived on PubMed Central

- yours - you keep the copyright
BioMedcentral 